\title{
Economics
}

\section{Study on the Implementation Mechanism of Symbiosis Theory Used in Economic Field}

\author{
Xingang Weng, Xiaohua Lv, Xiaorong Li \\ Logistics Institute, Beijing Wuzi University, Beijing, China \\ Email address: \\ wengxingang@263.net (Xingang Weng),771971104@qq.com (Xiaohua Lv),xhalw2012@163.com (Xiaohua Lv), \\ lixiaorong426@icloud.com (Xiaorong Li)
}

\section{To cite this article:}

Xingang Weng, Xiaohua Lv, Xiaorong Li. Study on the Implementation Mechanism of Symbiosis Theory Used in Economic Field. Economics. Vol. 5, No. 4, 2016, pp. 56-63. doi: 10.11648/j.eco.20160504.11

Received: July 22, 2016; Accepted: August 1, 2016; Published: August 29, 2016

\begin{abstract}
Symbiosis refers to the close relationship between two or more than two different species. They interact each other in long-term and form a mutually beneficial coexistence. The symbiosis theory is not only widely used in animal sciences and botany, but also has been extended to other disciplines. However, there are some defects in the process of the application of symbiosis theory in the field of economy. On one hand, this paper analyzes symbiotic interaction relationship: the patterns of symbiotic behavior and the patterns of symbiotic organization. On the other hand, this essay summarizes the main analysis method of symbiosis theory, and draws the logic diagram. By establishing a completer system of symbiosis theory, to help to promote the effective application of symbiosis method for further study in China.
\end{abstract}

Keywords: Symbiosis, Symbiotic System, Symbiosis Theory, Co-evolution

\section{Introduction}

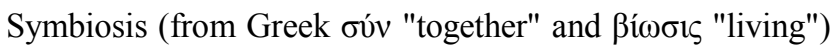
refers to the close relationship between two or more than two different species. They interact each other in long-term and form a mutually beneficial coexistence. [1] In 1877 Albert Bernhard Frank used the word symbiosis to describe the mutualistic relationship in lichens. In 1879, the German mycologist Heinrich Anton de Bary defined it as "the living together of unlike organisms." [2] [3] The definition of symbiosis has varied among scientists. Some believe symbiosis should only refer to persistent mutualisms, while others believe it should apply to any type of persistent biological interaction. After nearly 140 years of debate, current biology and ecology textbooks now use the latter "de Bary" definition or an even broader definition (where symbiosis means all species interactions). [4] The symbiotic phenomenon is a common phenomenon in nature, in the evolutionary history, its significance is equal to survival of the fittest.

The early studies of symbiosis theory mainly are botany and biology, the research methods mainly are qualitative analysis, the research content is mainly about the material and energy interaction of animals and plants, or about their influence degree. The biologist Lynn Margulis, famous for her work on endosymbiosis, contends that symbiosis is a major driving force behind evolution. She considers Darwin's notion of evolution, driven by competition, to be incomplete and claims that evolution is strongly based on co-operation, interaction, and mutual dependence among organisms. [5] From then on, this unique research method is not only widely used in the biological world, but also quickly spread to other fields of science, such as medicine, architecture, art, economy and so on.

\section{Concept of Symbiosis}

Symbiosis refers to the close relationship between two or more than two different species. The theory of symbiosis is a new frontier theory, which is permeated in the fields of medicine, agriculture, ecological civilization, architecture, art, economy, politics, culture and so on. It is a new world outlook, values and methodology. In 1980s, the famous Japanese architect Kisho Kurokawa put forward Philosophy of Symbiosis. Symbiosis concept includes not only in the field of art, culture, politics, science and technology, it is also involves to the human and the natural symbiosis, the art and science of symbiosis, rational and perceptual symbiosis, traditional and cutting-edge technology of symbiosis, regional and global symbiosis, history and future of symbiosis, different in 
symbiosis, urban and rural areas of symbiosis, the sea and the forest of symbiosis, abstract and symbol of symbiosis, the part and the whole in a symbiosis, the flesh and the spirit of symbiosis, conservative and innovation of symbiosis, development and protection of the symbiotic and other symbiotic in different levels. [6] Kisho Kurokawa predicted that the life time will turn from binary to the symbiosis, and the symbiotic order is the new world order in twenty-first Century. Yuan Chunqing (1998) defined the symbiotic unit as dependence relation according to some symbiotic pattern in the symbiotic environment. The core of which is a win-win and coexistence relationship. Yuan Chunqing first used symbiotic methods completely to study Chinese small economy. [7] Cheng Da Tao (2003) first analysis enterprise cluster from the microscopic point of view systematically by using symbiotic theory, he found that enterprise cluster symbiosis is beneficial to reduce the enterprise cluster market transaction cost and internal cost management, and improving the overall competitiveness of the cluster. [8] Hu Shoujun (2006) pointed that the symbiosis is the basic way of human living, on the one hand, human use symbiotic as the guiding ideology to optimize the allocation of resources, on the other hand, people have to manage themselves and establish the favorable natural feedback mechanism in order to achieve the harmony of man, nature and society. [9] Wang Shaoping, Ling Lan (2012) pointed that ecological industrial symbiosis is an economic group combined by agglomerated enterprises engaging in related industry. The organization structure of the symbiotic body determines the stability of the symbiosis and the behavior of the enterprise, and the behavior of the enterprise can affect the stability of the symbiosis. The industrial symbiosis organization structure forms in the process of the development of the symbiosis gradually, which will affect the behavior of the enterprises in the symbiotic relationship and the relationship between competition and cooperation in turn. [10] Combined with the existing research, the symbiotic nature is summed up as the following five aspects:

\subsection{Symbiosis Is a Kind of Self-Organization Phenomenon}

The phenomenon of symbiosis includes the symbiosis of nature and the phenomenon of human society. Symbiotic self-organization is a system, which can eliminate fault and improve the function by itself to a certain extent. In this system, the symbiotic unit can exchange information and energy and information. The symbiotic system has the characteristics of openness, hierarchy, nonlinearity, non-balance and life. [11]

\subsection{Symbiotic System Contains Three Elements}

Symbiotic system consists of three elements, symbiotic unit, symbiotic model and symbiotic environment. The symbiotic unit is the basic energy production and exchange unit of the symbiotic system. Symbiosis mode is the key of symbiotic system, which is the way of interaction and combination of symbiotic units in symbiotic system. Symbiotic environment is the external conditions for the existence and continuous existence of symbiotic units and symbiotic relationships.

\subsection{The Essence of Symbiotic Relationship Is Competition and Cooperation}

In the symbiotic system, there are both confliction and competitiveness behavior, and there are mutual tolerance and cooperative evolutionary behavior. The nature of the symbiotic relationship is to produce creative cooperation in the process of competition. It is the win-win situation that "the all things are not harmful to the world, but not to the contrary." The rational symbiotic result is forming the symmetric and mutually beneficial symbiotic relationship through competition and cooperation.

\subsection{New Energy Is Produced in the Process of Symbiosis}

Producing new energy is one of the essential features of symbiosis in the symbiotic process. Symbiosis energy has a variety of forms of expression. Mainly for individuals or organizations raising or breeding capacity enhancement because of the symbiotic relationship in nature. In the economic field, mainly for enterprises or clusters because of the coexistence of relations and enhance ability to resist external risks and enhance the capacity of overall economic efficiency. The level and effect of symbiotic units, symbiotic patterns, and environmental interactions in a symbiotic relationship, generally expressed as the value added of the symbiotic energy.

\subsection{The General Trend of Symbiotic System Is Co-evolution}

Evolution is the general direction of the development of symbiotic system. [14] The establishment of any symbiotic system is to enhance the survival ability and development ability of each symbiotic unit, and the enhancement of this kind of ability will promote the development of the symbiotic relationship to a more advanced form. So it can be said that the ideal evolution path of symbiotic system is from the lower stage to the advanced stage development.

Through the above analysis, it can be learned that the symbiotic connotation has certain relationship. The specific performance is shown in figure 1:

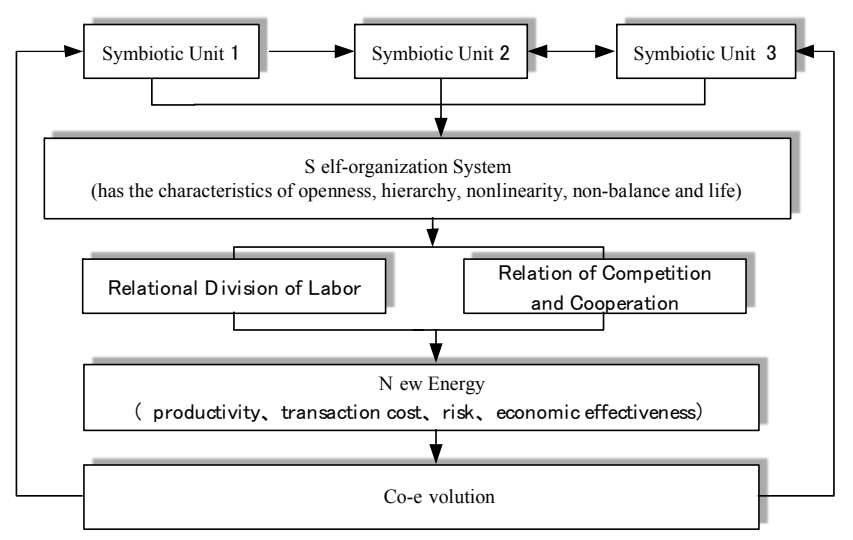

Figure 1. Association graph of symbiotic meaning. 


\section{Three Key Elements of Symbiotic System}

\subsection{Symbiotic Unit}

Symbiotic unit is the significant factor for the formation of a symbiotic relationship. It refers to the basic energy production and exchange unit that form the symbiotic or symbiotic relationship. [15] In the economic field, each enterprise is a symbiotic unit. In the process of research, the quality parameters and the image parameters are usually introduced to reflect the characteristics of the symbiotic units.

Quality parameters are the factors that determine the intrinsic nature of the symbiotic unit. The quality parameters of a symbiotic unit are varied, however, typically a unique quality parameter plays a key role in a certain period of time. This unique quality parameter is called as the main quality parameter. Image parameters refer to the factors that affect the external nature of symbiotic units. For instance, a logistics enterprise can be regarded as a symbiotic system, the delivery staff, dispatchers, managers, customer service staff and sales staff of the logistics enterprise can be regarded as five symbiotic units. Therefore, the professional ethics and ability can be regarded as the common quality parameters of the five symbiotic units, the enterprise culture can be regarded as the common image parameter of the the five symbiotic units.

\subsection{Symbiotic Model}

Symbiotic model, also named as symbiotic relationship, refers to the communicative forms among symbiotic units.
The symbiotic model not only reflects the interaction between the symbiotic units, but also reflects the intensity of the interaction. It not only reflects the exchange of material, energy and information between the symbiotic units, but also reflects the degree of the exchange. Different scholars have different classification of symbiotic model. For example, Caullery and Lewils defined symbiotic models included mutually beneficial symbiotic, living phenomenon, parasitic and other relationships between different organisms. Through the study of insects, O. Wilson Edward divided the symbiotic relationship into groups' parasitic, groups' commensalism and groups' mutualistic relationship. [16, 17] Yuan Chunqing divided symbiotic relationship into two types: the symbiotic behavior patterns and symbiotic organizational patterns. The symbiotic behavior patterns include parasitism, commensalism, asymmetric mutualistic relationship and symmetric mutualistic relationship; the symbiotic organizational patterns include point's symbiosis, intermittent symbiosis, consecutive symbiosis and integrative symbiosis [18] $\mathrm{Hu}$ Xiaopeng proposed three forms of symbiotic interaction: active, passive and followed. [19]

According to the existing researches, this paper firstly selects the interactive relationship, characteristics of symbiotic unit, characteristics of symbiotic energy and symbiotic effect to conduct a comparative analysis of symbiotic behavior patterns, as shown in table 1 . Then, selecting four indicators, interactive relationship, characteristics of symbiotic unit, characteristics of symbiotic energy and characteristics of symbiotic interface, to conduct a comparative analysis of symbiotic organizational patterns, as shown in table 2 .

Table 1. Symbiotic behavior patterns

\begin{tabular}{|c|c|c|c|c|}
\hline $\begin{array}{l}\text { Symbiotic } \\
\text { behavior } \\
\text { patterns } \\
\end{array}$ & Interaction & Characteristics of symbiotic units & $\begin{array}{l}\text { Characteristics of } \\
\text { symbiotic energy }\end{array}$ & Symbiotic effect \\
\hline Commensalism & Followed-passive & $\begin{array}{l}\text { (1) The morphological differences of } \\
\text { symbiotic units are obvious. } \\
\text { (2) The same kinds of units are close to } \\
\text { each other, the different kinds of units } \\
\text { exist bilateral relationship. }\end{array}$ & $\begin{array}{l}\text { (1) New energy is } \\
\text { generated. } \\
\text { (2) One party gets all the } \\
\text { new energy, the other party } \\
\text { does not get any new } \\
\text { energy. }\end{array}$ & $\begin{array}{l}\text { (1) Commensal relationship is } \\
\text { beneficial to one party, while the other } \\
\text { party gets no benefit. } \\
\text { (2) It is conducive to the evolution of } \\
\text { the profit side. }\end{array}$ \\
\hline $\begin{array}{l}\text { Asymmetric } \\
\text { mutualistic } \\
\text { relationship }\end{array}$ & Active- followed & $\begin{array}{l}\text { (1) The morphological differences of } \\
\text { symbiotic units are little. } \\
\text { (2) The proximity of the same kinds' units } \\
\text { exist largely difference, the different kinds } \\
\text { of units exist bilateral relationship. }\end{array}$ & $\begin{array}{l}\text { (1) New energy is } \\
\text { generated. } \\
\text { (2) One party gets the most } \\
\text { of the new energy, the other } \\
\text { party gets few new energies. }\end{array}$ & $\begin{array}{l}\text { (1) The asymmetric mutualistic } \\
\text { relationship is beneficial to both } \\
\text { parties, but the benefits exist } \\
\text { difference. } \\
\text { (2) It is conducive to both parties to } \\
\text { evolve, but the synchronization of } \\
\text { evolution exists a difference. }\end{array}$ \\
\hline $\begin{array}{l}\text { Symmetric } \\
\text { mutualistic } \\
\text { relationship }\end{array}$ & Active-active & $\begin{array}{l}\text { (1) The morphological differences of } \\
\text { symbiotic units hardly exist. } \\
\text { (2) The proximity of the same kinds' units } \\
\text { is similar or identical, } \\
\text { the different kinds of units exist bilateral } \\
\text { relationship. }\end{array}$ & $\begin{array}{l}\text { (1) New energy is } \\
\text { generated. } \\
\text { (2) The two parties get the } \\
\text { equivalent new energy. }\end{array}$ & $\begin{array}{l}\text { (1) The symmetric mutualistic } \\
\text { relationship is beneficial to both } \\
\text { parties, and the profits are equal; } \\
\text { (2) It is conducive to both parties to } \\
\text { evolve, but the synchronization of } \\
\text { evolution keeps consistent. }\end{array}$ \\
\hline
\end{tabular}


Table 2. Symbiotic organizational patterns.

\begin{tabular}{|c|c|c|c|c|}
\hline $\begin{array}{l}\text { Symbiotic } \\
\text { organizational patterns }\end{array}$ & Interaction & Characteristics of symbiotic units & $\begin{array}{l}\text { Characteristics of } \\
\text { energy exchange }\end{array}$ & $\begin{array}{l}\text { Characteristics of symbiotic } \\
\text { interface }\end{array}$ \\
\hline Point's symbiosis & Random & $\begin{array}{l}\text { (1) In a certain period, the interaction of } \\
\text { symbiotic units is one-time. } \\
\text { (2) Symbiotic elements interact in a certain area. }\end{array}$ & $\begin{array}{l}\text { Exchange on the one } \\
\text { side }\end{array}$ & $\begin{array}{l}\text { (1) single Medium. } \\
\text { (2) The interfaces are unstable. }\end{array}$ \\
\hline Intermittent symbiosis & Random & $\begin{array}{l}\text { (1) In a certain period, the interaction of } \\
\text { symbiotic units is many times. } \\
\text { (2) The interaction of a symbiotic unit on one or a } \\
\text { few aspects. }\end{array}$ & $\begin{array}{l}\text { Minority } \\
\text { communication }\end{array}$ & $\begin{array}{l}\text { (1) Minority medium. } \\
\text { (2) The interfaces are unstable. }\end{array}$ \\
\hline Consecutive symbiosis & Inevitable & $\begin{array}{l}\text { the interaction of symbiotic units is consecutive. } \\
\text { Interaction } \\
\text { (2) Symbiotic elements interact in a number of } \\
\text { aspects. }\end{array}$ & $\begin{array}{l}\text { Many-sided } \\
\text { communication }\end{array}$ & $\begin{array}{l}\text { (1) Many mediums, and the } \\
\text { mediums compensate each other. } \\
\text { (2) The interfaces are stable. }\end{array}$ \\
\hline Integrative symbiosis & Inevitable & $\begin{array}{l}\text { (1) In a certain period, nature and function is } \\
\text { unify between the symbiotic units. } \\
\text { (2) The symbiotic unit has a full range of } \\
\text { interactions. }\end{array}$ & $\begin{array}{l}\text { All round } \\
\text { communication }\end{array}$ & $\begin{array}{l}\text { (1) Mediums are diversified, and } \\
\text { existing a core medium; } \\
\text { (2) The interfaces are really } \\
\text { stable. }\end{array}$ \\
\hline
\end{tabular}

\subsection{Symbiotic Environment}

The symbiotic environment is the carrier for the existence and continuation of symbiotic units and symbiotic relationships. In general, the sum of all the factors other than the symbiotic elements forms a symbiotic environment. In the economic field, the symbiotic environment of enterprises includes market environment and social environment. According to the influence of environmental factors on the evolution direction of the symbiotic system, the symbiotic environment is usually divided into three types: the positive environment, the neutral environment and the reverse environment. As is shown in table 3.

Table 3. Symbiotic environment.

\begin{tabular}{ll}
\hline $\begin{array}{l}\text { Symbiotic } \\
\text { environment }\end{array}$ & Meaning \\
\hline $\begin{array}{l}\text { Positive } \\
\text { environment }\end{array}$ & $\begin{array}{l}\text { Environment for promoting the development of } \\
\text { symbiotic unit evolution and symbiotic relationship. } \\
\text { Neutral } \\
\text { environment }\end{array}$ \\
$\begin{array}{l}\text { Environment for the evolution of symbiotic unit and } \\
\text { the development of symbiotic relationship. } \\
\text { environment }\end{array}$ & $\begin{array}{l}\text { Environment hampers the development of symbiotic } \\
\text { unit evolution and symbiotic relationship. }\end{array}$ \\
\hline
\end{tabular}

\section{The Basic Principles of Symbiosis}

The basic principle of symbiosis reveals the essential rules of formation and development of symbiotic system. The basic principle of symbiosis mainly includes the principle of quality parameter compatibility, the principle of symbiotic energy generation, the selection principle of symbiotic interface and the principle of symbiotic evolution. [21]

\subsection{The Principle of Quality Parameter Compatibility}

As the intrinsic property of the expression of the symbiotic unit, the quality parameter is compatible with the expression of the quality parameters. For example, enterprises A and B is the relationship between supply and demand, for a certain product, the input of enterprise $A$ and the output of enterprise $\mathrm{B}$ would have a certain corresponding relationship. The mathematical expression of quality parameter compatibility is as follows.

If $Z_{A}=f\left(Z_{B}\right)$, then $A$ and $B$ may form a symbiotic relationship, in which $f\left(Z_{B}\right)$ can be random function, discontinuous function or continuous function.

If $\mathrm{f}\left(Z_{B}\right)$ is Random function, then $\mathrm{A}$ and $\mathrm{B}$ is generally easy to form the point's symbiotic mode.

If $\mathrm{f}\left(Z_{B}\right)$ is discontinuous function, then $\mathrm{A}$ and $\mathrm{B}$ is generally easy to form intermittent symbiosis model.

If $\mathrm{f}\left(Z_{B}\right)$ is continuous function, then $\mathrm{A}$ and $\mathrm{B}$ is generally easy to form a consecutive symbiotic model or an integrative symbiotic model.

Therefore, the possibility of the formation of the symbiotic relationship and the pattern of the symbiotic relationship are compatible.

The principle of quality parameter compatibility reveals the basic rules of the formation and development of the symbiotic model. On the one hand, it provides the basis for our objective understanding and using of the natural symbiotic system; on the other hand, it points out the direction for our scientific design and construction of social symbiotic system. Therefore, it is very important to use the principle of quality parameter to promote the construction of the symbiotic system.

\subsection{The Principle of Symbiotic Energy Generation}

The symbiotic units will produce new energy in the process of interaction, this new energy also known as symbiotic energy. Symbiotic energy has a variety of forms of expression. In nature, it is generally expressed as an individual or a population that enhances the ability to survive or reproduce because of the symbiotic relationship. In the field of social and economic performance of enterprises or organizations to enhance the overall competitiveness of the symbiotic relationship, enhance the ability to resist external risks, expand the scale of operations and increase economic benefits, etc. Assuming the total energy of the symbiotic system is E, As for a two-dimensional symbiotic system, if there are symbiotic units $\mathrm{A}$ and $\mathrm{B}, E_{A}$ and $E_{B}$ represent the energy of $\mathrm{A}$ and $\mathrm{B}$, respectively, $E_{m}$ represents the new 
energy that generated by A and B' mutual relationship, the mathematical expression of the symbiotic system energy can be expressed as:

$$
\mathrm{E}=E_{A}+E_{B}+E_{m}
$$

The principle of energy generation shows that the total energy of a symbiotic system depends on the independent energy of each symbiotic unit and the energy that arising from their interaction. The formation of symbiotic energy is mainly the result of the interaction between the elements of the symbiotic units. In the symbiosis theory, the general use of symbiotic energy value added to show the symbiotic relationship of symbiotic units, symbiotic model and symbiotic environment interaction efficiency. The symbiotic energy is dynamic, and the changes of the symbiotic energy affect the direction and extent of the evolution of the symbiotic system.

Overall, the principle of symbiotic energy generation is an effective tool to identify the level and efficiency of symbiotic system operation. By using this principle, it will be advantageous to promote the coordinated evolution of symbiotic units, promote the benign development of symbiotic relationship and improve the symbiotic environment.

\subsection{The Selection Principle of Symbiotic Interface}

Symbiotic interface refers to the dependent medium of the information exchange, energy transfer, material exchange, cooperation and competition, symbiotic order and so on. The symbiotic interface is divided into a single medium symbiotic interface and a multi medium symbiotic interface. In the social symbiotic system, there are often multiple symbiotic relationships, so the multi medium symbiotic interface is more common. The choice of the symbiotic interface not only determines the quantity and quality of the symbiotic unit, but also determines the capacity of the production and reproduction of the symbiotic energy. Therefore, it is great significance to grasp the principle of the selection of the symbiotic interface.

The formation of the symbiotic interface is mainly based on the nature and interaction model of the symbiotic units. In the selection process of symbiotic units, the main basis of the degree of information mastery. Under the condition of complete information, the non-competitive selection method is generally adopted in the symbiotic unit. The non-competitive selection method mainly includes the choice of the rule of closeness degree and the choice of the degree of association rule in two ways. Under the condition of incomplete information, the symbiotic unit generally use the competitive selection mode, namely through all candidate symbiotic units compete with each other, and then chooses the best. Competitive choice is also known as the law of elimination. The choice of symbiotic units reveals the relationship between information condition and competition. The more complete information is, the more effective the non -competitive selection is, the less complete information is, and the more effective the competitive selection is.
On the whole, the symbiotic interface selection principle reflects the relationship between symbiotic system and symbiotic environment. The optimal development mode of the symbiotic system should be in the process of symbiosis, which only consumes the minimum internal energy to obtain the maximum overall symbiotic energy. Once the symbiotic interface is formed, it has its own characteristics and operation mode. Therefore, the symbiotic interface has the characteristics of relative stability and non-replication. By using the symbiotic interface selectivity principle, it is beneficial to promote the formation and development of the symbiotic system.

\subsection{The Principle of Symbiotic Evolution}

The principle of symbiotic system evolution reveals the essence of symbiotic evolution. In the process of symbiotic system evolution, different symbiotic units often have different status and function. The formation of a symbiotic system, it is always from the lower stage to the advanced stage of evolution. In a symbiotic system, if $\alpha$, $\beta$ and $\gamma$ are three factors affecting the evolution of the system, The evolution of the symbiotic system $\mathrm{Z}$ can be expressed as a mathematical expression,

$$
\mathrm{Z}=f(\alpha, \beta, \gamma)
$$

In the principle of symbiotic system evolution, all of the symbiotic relationships are formed and grow in the non-symmetric and mutually beneficial relationship, and eventually evolved into a symmetrical and mutually beneficial relationship. In all symbiotic systems, symmetric mutualistic relationship is theoretically the most stable model of the highest efficiency. In the system of asymmetric mutualistic relationship, there are the key factors that affect the efficiency of the symbiotic system, the smaller effect of the key factors to the asymmetric distribution, the more energy will be generated. In the system of symmetric mutualistic relationship, the ability of each symbiotic unit has been the ultimate play. They interact to produce the maximum energy of these new energies was a symbiotic unit share.

The evolution principle of the symbiotic system is one of the cores of the symbiotic theory. It reveals the basic rules of the symbiotic evolution. The principle of symbiotic system evolution is a favorable tool for us to understand the natural symbiotic system and social symbiotic system, which is of great significance to promote the prosperity and development of the symbiotic system.

\section{The Analytic Methods of Research on Symbiotic Theory}

\subsection{The Basic Analytic Methods of Research on Symbiotic Theory}

Using symbiotic theory, two main methods in the analysis of practical problems, they are symbiotic model analytic method and symbiotic network analytic method. These two 
methods are sometimes carried out individually, sometimes simultaneously. In general, they constitute the main research of symbiotic theory in China.

\subsubsection{Symbiotic Model Analytic Method}

At present, there is abundant literature about the symbiotic models. Symbiotic model analytic method refers to the research on the symbiotic system or its formation and development, on the researching of symbiotic model, the scholars try to find the essential rules of the symbiotic system, and try to determine the direction of symbiotic system and accelerate the evolution of symbiotic ecosystems. One one hand, there is abundant theoretical research on symbiotic mode, such as above section 3.2. On the other hand, there is abundant empirical research on symbiotic mode. such as Yang Chunhe (2009) used the symbiosis theory to analyze the logistics industry cluster. [22] He divided the evolution into symbiotic model and symbiotic behavior patterns firstly, and then put forward the corresponding implementation path, as is shown in figure 2.

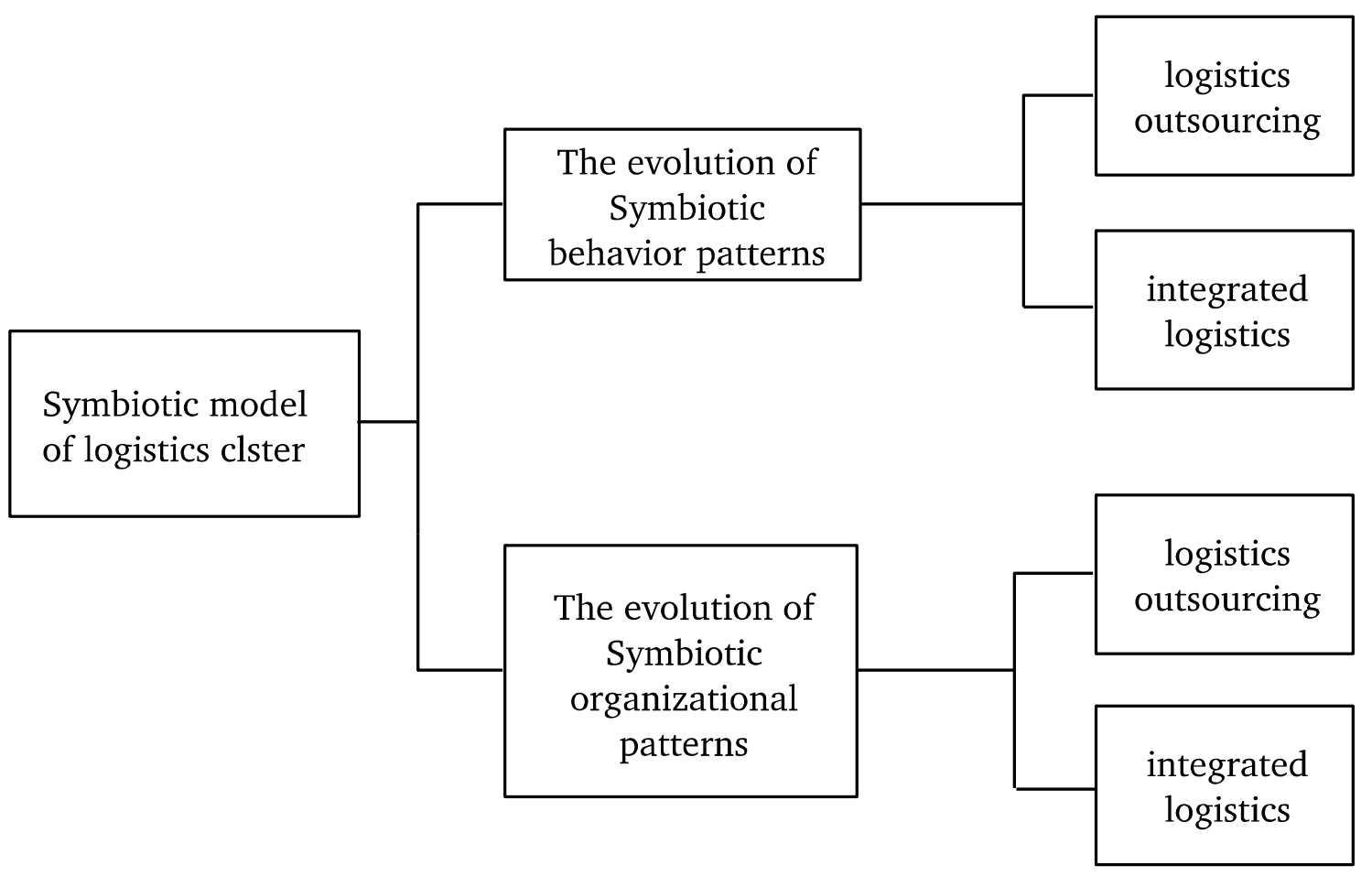

Figure 2. Logistics cluster symbiotic evolution model.

\subsubsection{Symbiotic Network Structure Analytic Method}

Symbiotic network structure analytic method refers to through of symbiotic unit environment established with some structural features of the network analysis, and draws the symbiosis network of local and global features. Symbiotic model analytic method provides the direction of design and construction of symbiotic system. Wang Shaoping, Ling Lan (2012) divided the content of industrial symbiosis network structure analysis into the following types, basing on to the network of industry symbiosis structure characteristics and symbiosis pattern clustering analysis, basing on to the network of industrial symbiosis structure characteristics and symbiosis pattern clustering analysis and industrial symbiosis network stability analysis. Symbiosis network structure characteristics including network symbiotic relationship type and network size (number of nodes and the number of relations), agglomeration degree of network density and clustering coefficient), information transmission speed (characteristic path length), center, node influence and network complexity etc. Symbiosis network symbiosis pattern clustering analysis refers to network density and clustering coefficient, average degree and characteristic path length, centrality index and all structure characteristics value factor cluster analysis, to make the comprehensive evaluation of clustering results. [23]

Symbiotic network analysis is an effective combination of qualitative research and quantitative research, and the results of the analysis are the quantitative criteria for the classification of industrial symbiosis network. It is of great significance to improve the analysis of the symbiotic network to promote the symbiotic theory.

\subsection{The Logical Analytic Methods of Research on Symbiotic Theory}

The basic logic of the symbiotic theory is based on the symbiosis, identifying and confirming all the logical nodes of the symbiotic system, as well as the logical judgment relation among the nodes, so as to reveal the operation mechanism of the symbiotic system. Li Liangxian (2011) summed up 8 logical nodes forming a symbiotic system analysis. The basic logic of symbiosis theory is shown in figure 3. [24] 


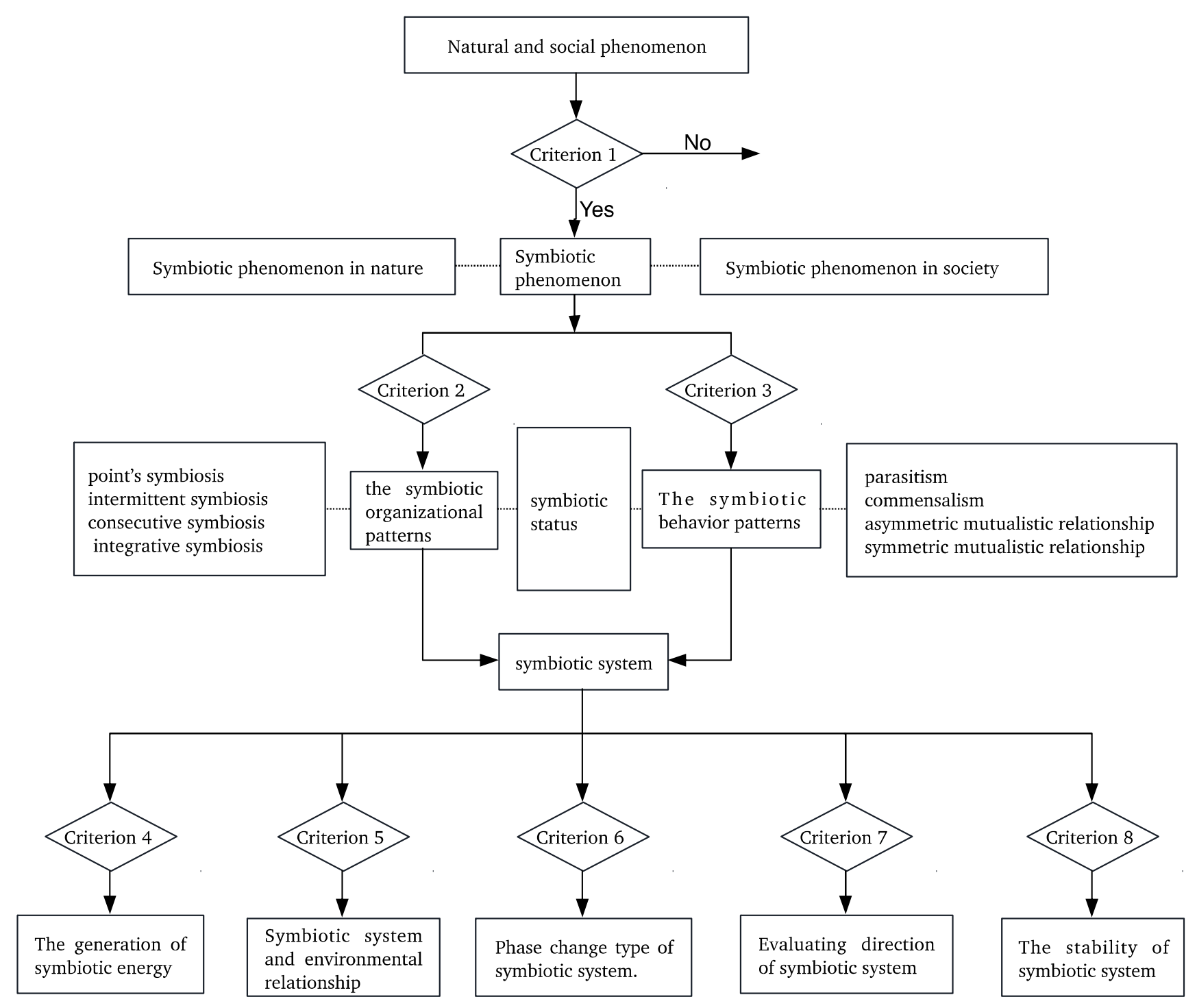

Figure 3. Basic logic of symbiosis analysis.

The 8 criterions is the logical basis for the analysis of symbiotic system.

Criterion 1. Compatibility of quality parameter. The possibility formation of the symbiotic relationship is compatible with the compatibility of the quality parameters, and there is a possibility to form a symbiotic relationship if there is only the existence of a quality parameter.

Criterion 2. The pattern of symbiotic organization. It is based on the degree of symbiotic organization to determine what kind of organizational pattern. The symbiotic organizational pattern includes point's and intermittent symbiosis, consecutive symbiosis and integrated symbiosis.

Criterion 3. The pattern of symbiotic behavior. It is based on the behavior of symbiotic units to determine the specific pattern of symbiotic phenomenon. The pattern of symbiotic behavior includes parasitism, commensalism, asymmetric mutualistic relationship and symmetric mutualistic relationship.

Criterion 4. The generation of symbiotic energy. The formation of symbiotic energy is the basic condition for the survival of the symbiotic system. It has a dynamic change, and the changes of the symbiotic energy affect the direction and extent of the evolution of the symbiotic system.

Criterion 5. Symbiotic system and environmental relationship. The symbiotic environment is the carrier of the symbiotic relationship, and it is all the external factors that affect the symbiotic unit. The symbiotic environment is mainly divided into three types, positive environment, neutral environment and reverse environment.

Criterion 6. Phase change type of symbiotic system. Phase change is the characteristic manifestation of the change of symbiotic system. Different reasons can form different types of phase transition.

Criterion 7. Evaluating direction of symbiotic system. The evolution principle reveals the nature of the symbiotic system evolution.

Criterion 8. The stability of symbiotic system. The symbiotic relationship has the characteristic of stability, and the symbiosis degree is the main parameter to judge the 
stability of the symbiotic system.

\section{Conclusion}

Symbiosis theory is a hot topic research fields in the current or the future. Combining with the existing related literature, this paper analyzed the related knowledge of symbiosis which including the origin of symbiosis and the basic meaning of symbiosis at first. Then the three elements of symbiotic system were introduced, which were symbiotic unit, symbiotic model and symbiotic environment. And then the basic principle of symbiosis was explained. They are respectively the principle of quality parameter compatibility, the principle of symbiotic energy generation, the selection principle of symbiotic interface and the principle of symbiotic system evolution. Finally, analytic methods of research on symbiotic theory was summarized, and the logic schematic diagram was compiled. Of course, this study is in the early stages of the symbiotic theory. The follow-up will be more in-depth exploration. Hoping this paper will play a positive role in promoting the symbiotic theory in the economic field.

\section{References}

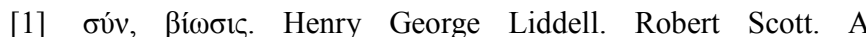
Greek-English Lexicon. revised and augmented throughout by. Sir Henry Stuart Jones. with the assistance of. Roderick McKenzie. Oxford. Clarendon Press. 1940.

[2] Surindar Paracer, Vernon Ahmadjian. Symbiosis: An Introduction to Biological Association [M]. Oxford University Press(2),2000.

[3] A. E. Douglas, symbiotic Interactions, Oxford University Perss, 1994,

[4] THE NEW OXFORD English-Chinese DICTIONARY [Z]. Shanghai: Shanghai Foreign Language Education Press, 2007.

[5] Lynn Margulis, Dorion Sagan. On the Origin of Mitosing Cells [J]. Journal of Theoretical Biology, March 1967, Volume 14 , Issue 3.

[6] Kurokawa (author), Tan Li (translator). Beijing: China Building Industry Press, 2009, vi.

[7] Yuan Chunqing. Symbiosis theory - discussion on small economy [M]. Beijing: Economic Science press, 1998.

[8] Cheng Cheng. Research on enterprise cluster organization based on Symbiosis Theory [D]. Hangzhou: Zhejiang University. 2003.
[9] Hu Shoujun. Social symbiosis theory [M]. Shanghai: Fudan University press, 2006

[10] Wang Shaoping, Ling LAN. Study on the structure characteristics of industrial symbiosis network [M]. Shanghai: Tongji University press, 2016

[11] Wang Zhenzhen, Bao Xinghua. Development status and application of industrial symbiosis theory [J]. East China economic management, 2012 (10), 131-136.

[12] Liu Rongzeng. Symbiosis theory and its role in the construction of a harmonious society $[\mathrm{J}]$. one hundred forum. 2006 (1), 126-127.

[13] Li Liangxian. Research on the development of small and medium sized enterprises based on Symbiosis Theory [M]. Beijing: economic management publishing house, 2011. 22-24.

[14] Study on formation mechanism and evolution of shipbuilding industry cluster based on Symbiosis Theory [D]. Nanjing: Nanjing University of Science and Technology, 2005

[15] Yang Chunhe. Modern logistics industrial cluster formation and evolution model [M]. Beijing: China Railway Publishing House, 2009, 52-59.

[16] V. Ahmdajina, Symbiosis: An Introduction Biological Association, University Press of New Englnad, 1986, 5-6, $42-45$.

[17] Mya, R·M- Stability and Complexity in Model Ecosystems. Princeton University Press, Princeton, NJ. 1989, 88-94.

[18] Yuan Chunqing. Symbiosis theory and Discussion on small economy [M]. Beijing: Economic Science Press, 1998

[19] Industrial Symbiosis: theoretical definition and its internal mechanism [J]. China's industrial economy, 2008 (9) 118-128.

[20] Ding Liyi. Symbiosis theory of the innovation of creative industry park mode [D]. Wuhan: Wuhan University of Technology. 2013.

[21] Rao Pingyang, Study on the formation and evolution of tourism industrial clusters in the perspective of symbiosis theory [D]. Xi'an: Northwest University. 2010.

[22] Yang Chunhe. The Formation and Evolution Mode of Modern Logistics Industrial Cluster [M]. Beijing: China Rail Way Publishing House, 2009, 52-118.

[23] Wang Shaoping, Ling LAN. Study on the structure characteristics of industrial symbiosis network [M]. Shanghai: Tongji University press, 2016. 48-75.

[24] Research on the development of small and medium sized enterprises based on the symbiosis theory [M]. Beijing: economic management publishing house, 2011. 27-29. 